\title{
QUEEN'S
UNIVERSITY
BELFAST
}

\section{Generation of high-energy-density ion bunches by ultraintense laser- cone-target interaction}

Yang, X. H., Yu, W., Xu, H., Zhuo, H. B., Ma, Y. Y., Zou, D. B., Yu, T. P., Ge, Z. Y., Yin, Y., Shao, F. Q., \& Borghesi, M. (2014). Generation of high-energy-density ion bunches by ultraintense laser-cone-target interaction. Physics of Plasmas, 21(6), [063105]. https://doi.org/10.1063/1.4882445

Published in:

Physics of Plasmas

Document Version:

Publisher's PDF, also known as Version of record

Queen's University Belfast - Research Portal:

Link to publication record in Queen's University Belfast Research Portal

\section{Publisher rights}

Copyright 2014 AIP Publishing LLC. This article may be downloaded for personal use only. Any other use requires prior permission of the author and the American Institute of Physics. The following article appeared in : Physics of Plasmas (1994-present) 21, 063105 (2014) and may be found at http://scitation.aip.org/content/aip/journal/pop/21/6/10.1063/1.4882445.

\section{General rights}

Copyright for the publications made accessible via the Queen's University Belfast Research Portal is retained by the author(s) and / or other copyright owners and it is a condition of accessing these publications that users recognise and abide by the legal requirements associated with these rights.

Take down policy

The Research Portal is Queen's institutional repository that provides access to Queen's research output. Every effort has been made to ensure that content in the Research Portal does not infringe any person's rights, or applicable UK laws. If you discover content in the Research Portal that you believe breaches copyright or violates any law, please contact openaccess@qub.ac.uk. 


\section{AIP | Physics of \\ Plasmas}

\section{Generation of high-energy-density ion bunches by ultraintense laser-cone-target interaction}

X. H. Yang, W. Yu, H. Xu, H. B. Zhuo, Y. Y. Ma, D. B. Zou, T. P. Yu, Z. Y. Ge, Y. Yin, F. Q. Shao, and M.

Borghesi

Citation: Physics of Plasmas (1994-present) 21, 063105 (2014); doi: 10.1063/1.4882445

View online: http://dx.doi.org/10.1063/1.4882445

View Table of Contents: http://scitation.aip.org/content/aip/journal/pop/21/6?ver=pdfcov

Published by the AIP Publishing

\section{Articles you may be interested in}

High energy density micro plasma bunch from multiple laser interaction with thin target

Appl. Phys. Lett. 104, 024105 (2014); 10.1063/1.4858956

Preplasma effects on the generation of high-energy protons in ultraintense laser interaction with foil targets Phys. Plasmas 20, 123105 (2013); 10.1063/1.4843975

High-density highly collimated monoenergetic $\mathrm{GeV}$ ions from interaction of ultraintense short laser pulse with foil in plasma

Phys. Plasmas 17, 043110 (2010); 10.1063/1.3385444

Enhancement of high-energy ion generation by preplasmas in the interaction of an intense laser pulse with overdense plasmas

Phys. Plasmas 11, 1726 (2004); 10.1063/1.1650844

High energy proton acceleration in interaction of short laser pulse with dense plasma target

Phys. Plasmas 10, 2009 (2003); 10.1063/1.1556298

\section{AIP $\mid$ chaos \\ CALL FOR APPLICANTS \\ Seeking new Editor-in-Chief}




\title{
Generation of high-energy-density ion bunches by ultraintense laser-cone-target interaction
}

\author{
X. H. Yang, ${ }^{1}$ W. Yu, ${ }^{2}$ H. Xu, ${ }^{3, a)}$ H. B. Zhuo, ${ }^{1, b)}$ Y. Y. Ma, ${ }^{1}$ D. B. Zou, ${ }^{1}$ T. P. Yu, ${ }^{1}$ Z. Y. Ge, ${ }^{1}$ \\ Y. Yin, ${ }^{1}$ F. Q. Shao, ${ }^{1}$ and M. Borghesi ${ }^{4,5, c)}$ \\ ${ }^{1}$ College of Science, National University of Defense Technology, Changsha 410073, China \\ ${ }^{2}$ Shanghai Institute of Optics and Fine Mechanics, Chinese Academy of Sciences, Shanghai 201800, China \\ ${ }^{3}$ State Key Laboratory of High Performance Computing, National University of Defense Technology, \\ Changsha 410073, China \\ ${ }^{4}$ School of Mathematics and Physics, Queen's University of Belfast, Belfast BT7 1NN, United Kingdom \\ ${ }^{5}$ Institute of Physics of the ASCR, ELI-Beamlines Project, Na Slovance 2, 18221 Prague, Czech Republic
}

(Received 22 September 2013; accepted 27 May 2014; published online 11 June 2014)

A scheme in which carbon ion bunches are accelerated to a high energy and density by a laser pulse $\left(\sim 10^{21} \mathrm{~W} / \mathrm{cm}^{2}\right)$ irradiating cone targets is proposed and investigated using particle-in-cell simulations. The laser pulse is focused by the cone and drives forward an ultrathin foil located at the cone's tip. In the course of the work, best results were obtained employing target configurations combining a low- $\mathrm{Z}$ cone with a multispecies foil transversely shaped to match the laser intensity profile. (C) 2014 AIP Publishing LLC. [http://dx.doi.org/10.1063/1.4882445]

\section{INTRODUCTION}

Ion bunches driven by intense laser pulses are of great interest for potential applications in areas including ion driven fast ignition, ${ }^{1}$ medical therapy, ${ }^{2}$ and proton probing. ${ }^{3,4}$ Various ion acceleration mechanisms have been proposed and investigated in the past decades, such as target normal sheath acceleration (TNSA), ${ }^{5,6}$ laser breakout afterburner (BOA), ${ }^{7}$ and radiation pressure acceleration (RPA). ${ }^{8,9}$

TNSA is one of the most widely studied ion acceleration mechanisms both in numerical simulations and experiments and can accelerate ions to several tens of $\mathrm{MeV}$ by the sheath's electric field on the target's rear surface. Such a mechanism can be easily achieved in current laser facilities, although the energy spectra of the ions driven by TNSA are usually not mono-energetic but broad and exponentially decaying with energy. ${ }^{10,11}$ In addition, since the energetic ions are dependent on the hot electron characteristics, their density is usually far below the critical density. ${ }^{12,13}$ In contrast, RPA is much more favorable for obtaining quasimono-energetic and dense ion beams. In this mechanism, the ions are accelerated by an intense space-charge field created by the radiation pressure of the laser pulse, and, in an ideal case, they co-move with an electron layer thanks to the balance between the radiation pressure and the space-charge field. ${ }^{14}$ In order to reduce heating of the electrons, a circularly polarized laser pulse is usually employed by particlein-cell (PIC) simulations, which have demonstrated that RPA is a very efficient ion acceleration regime for an ultrarelativistic laser pulse interacting with thin foils. ${ }^{15-17}$ The energy of the ions accelerated by RPA is proportional to $(I \tau / \sigma)^{2}$ for a nonrelativistic laser pulse and to $I \tau / \sigma$ for the ultrarelativistic limit, ${ }^{18}$ where $I$ is the laser intensity, $\tau$ is the pulse duration, and $\sigma$ is the areal mass density of the foil.

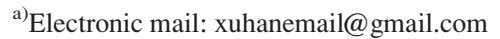

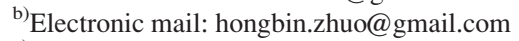

${ }^{\mathrm{c})}$ Electronic mail: m.borghesi@qub.ac.uk
}

The ion energy can reach $\mathrm{GeV} /$ nucleon energies for laser intensities in the $10^{22} \mathrm{~W} / \mathrm{cm}^{2}$ range. ${ }^{19}$ Such extremely high intensities are still out of reach for current lasers but could be achievable with the next generation of muti-petawatt (PW) facilities such as, for example, the Extreme Light Infrastructure (ELI). ${ }^{20}$ Recently, RPA mechanisms in multispecies targets have received particular attention, as simulations indicate that, in these targets, acceleration of the lighter ion species can acquire enhanced stability. ${ }^{21,22}$

In a number of TNSA experiments, flat-top conical targets have been used as a means to enhance the energy conversion in hot electrons using a linearly polarized laser pulse and have also been proposed as a possible way to enhance the laser intensity near the tip, in addition, to any external focusing. ${ }^{23-28}$ We investigate here by means of $2 \mathrm{D}$ PIC simulations whether such enhancement in intensity can be useful for RPA schemes, and for this purpose, we have tested a number of different target configurations irradiated by circularly polarized laser pulses at high intensities.

The configuration tested initially was that of a gold plasma cone and a multi-species slab foil composed by $\mathrm{C}^{6+}$ and $\mathrm{Cu}^{24+}$, as shown in Fig. 1(a) and used, for example, in Ref. 29. We see that this configuration does not lead to stable RPA conditions, but instead the foil is quickly fragmented by the laser pulse, which propagates through it, pushing sideways the remaining plasma clumps. The carbon ion energy spectrum is complex showing several peaks at low energies, and a monotonously decreasing distribution in the high energy end above $500 \mathrm{MeV}$.

In an attempt to improve the stability of the foil acceleration, we have then run simulations by varying the composition of the target, namely, by using (1) a lower density, hydrogen plasma cone instead of the gold plasma cone and (2) a Gaussian-shaped foil, thicker at the center and thinner at the periphery, instead of a slab foil, as pictured in Fig. 1(b). In this case, the ion layer does not fragment under the pressure of the radiation, but it is effectively accelerated as a 
(a)

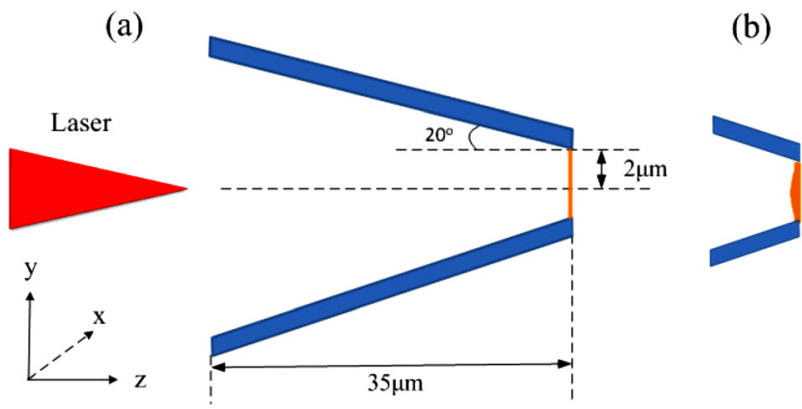

FIG. 1. Simulation setup: The cone target (blue) having a thickness of $1.5 \lambda_{0}$ and a half-cone-angle of $20^{\circ}$ is located between $z=5 \lambda_{0}$ and $40 \lambda_{0}$ in the simulation box. The inner radius of the cone tip is $2 \lambda_{0}$. (a) and (b) The cases with a slab cone tip and with a Gaussian-shaped cone tip, respectively. The target is irradiated by a circularly polarized laser pulse (red) from the left boundary.

whole along the laser axis. In these simulations, the laser pulse is seen to break through the H-plasma cone wall or from the thinner foil periphery. As a result, the $\mathrm{C}^{6+}$ ion layer is accelerated along the laser axis like a bullet and the laser radiation transmitted at the edges can wrap around and help compress the ion layer, as observed in Ref. 30. In our simulations, using a $10^{21} \mathrm{~W} / \mathrm{cm}^{2}$ laser, a quasi-mono-energetic peak at $\sim 790 \mathrm{MeV}$ appears in the energy spectrum of $\mathrm{C}^{6+}$ ions, which contains about $31 \%$ of the total $\mathrm{C}^{6+}$ ion energy. The maximum energy density of the resulting ion bunch is $\sim 7 \times 10^{10} \mathrm{~J} / \mathrm{cm}^{3}$. It seems therefore that such an approach may, in principle, be of interest for achieving the radiation pressure drive of high energy ion beams with reachable laser intensities in the near future.

\section{SIMULATION MODEL}

The aim of this study is to propose a scheme for efficient acceleration of the ions located at the tip of the conical target under the effect of intense radiation pressure. The simulations are performed using the relativistic 2D3V PIC code LAPINE. ${ }^{31,32}$ Our initial target set-up contains a cone and a foil at the tip, as depicted in Fig. 1. A circularly polarized laser pulse is incident normally from the left boundary. A cone with a half-aperture of $20^{\circ}$ is located initially between $z=5 \lambda_{0}$ and $40 \lambda_{0}$, where $\lambda_{0}=1 \mu \mathrm{m}$ is the laser wavelength. The thickness of the cone wall is $1.5 \lambda_{0}$. For the first configuration, the cone plasma consists of an initially neutral mixture of electrons and $\mathrm{Au}^{40+}$ ions with mass $m_{A u}=197 m_{p}$, where $m_{p}=1836 m_{e}$ is the proton mass. The density of the $\mathrm{Au}^{40+}$ is set to $5 n_{c}$, corresponding to an electron density of $200 n_{c}$, where $n_{c}=1.12 \times 10^{21} \mathrm{~cm}^{-3}$ is the critical density. The thickness of the attached foil is set to be $0.14 \lambda_{0}$ and its radius is $2 \lambda_{0}$. In order to facilitate RPA, ${ }^{21,22}$ the foil at the cone tip is composed of $\mathrm{C}^{6+}$ with mass $m_{C}=12 m_{p}$ and $\mathrm{Cu}^{24+}$ with mass $m_{C u}=63.5 m_{p}$, whose densities are both set to $8 n_{c}$, and the electron density is $240 n_{c}$. Using such a multispecies foil, the lower- $Z$ ion species can acquire supplementary electrons from those associated with the high- $Z$ ion species. This can preserve a locally quasi-neutral spacecharge and lead to a stable RPA. ${ }^{22}$ The simulation box is $70 \lambda_{0} \times 40 \lambda_{0}$ with $3500 \times 2000$ cells. Each cell contains 49 numerical macroparticles per species in the simulations. In order to resolve the Debye length, the initial temperature of both electrons and ions is set to $1 \mathrm{keV}$. It is noted that, by using such an initial temperature, the foil expands to twice the initial thickness, with a pedestal electron density of $\sim 12 n_{c}$, and the maximum density of the electrons becomes $\sim 200 n_{c}$ before the laser pulse impacts it. The laser pulse has a trapezoidal temporal profile, which rises up linearly for a duration $1 \mathrm{~T}_{0}$ to the peak intensity, stays at constant intensity for $10 \mathrm{~T}_{0}$, then decreases linearly over a duration $1 \mathrm{~T}_{0}$, where $T_{0} \sim 3.3 \mathrm{fs}$ is the laser cycle. The dimensionless maximum amplitude of the laser electric field is $a_{0}=e E_{0} / m_{e} c \omega_{0}=25$, where $E_{0}$ and $\omega_{0}$ are the electric field and frequency of the laser pulse, respectively. The spatial profile of the laser pulse is Gaussian with a spot radius of $13.5 \lambda_{0}$, corresponding to a laser power of $9.8 \mathrm{PW}$. Laser pulses of high contrast in excess of $10^{10}$ are available, ${ }^{33-38}$ allowing good target integrity to be maintained before the main pulse irradiates the cone tip. Thus, the effect of a characterized laser pre-pulse is not intentionally simulated here. However, the thermal expansion of the $1 \mathrm{keV}$ initial condition target is akin to the effect of an uncharacterized pre-pulse to the main pulse, which is fortuitous as even the highest contrast lasers still have $10 \mathrm{~s}-100 \mathrm{~s}$ of ps of pre-pulse. The time step is $0.007 \mathrm{~T}_{0}$. The simulations are run until $150 \mathrm{~T}_{0}$. For both the transverse and longitudinal boundaries, absorbing boundary conditions are used for the fields and particles.

\section{RESULTS AND DISCUSSION}

In case $\mathrm{A}$, the target is composed of a gold plasma cone wall and a multi-species slab foil at the tip. Characteristics of the laser pulse and of the target for case A are presented in Table I. Figure 2 shows the distribution of the transverse electric field $\left(E_{y}\right)\left(\right.$ a) at $t=55 T_{0}$ and the charge density of $\mathrm{C}^{6+}$ ions $\left(Z_{C} n_{C}\right)$ (b) at $t=70 T_{0}$ for case $\mathrm{A}$. It is seen that the laser pulse is focused due to the nonlinear interaction between the laser pulse and the gold cone. ${ }^{25}$ To avoid the influence of the reflected laser pulse from the cone tip and obtain enhancements of the laser intensity by the cone, a gold cone without a cone tip is employed. It is found that the

TABLE I. Characteristics of the laser and of the target employed in the cases A, B, and C, where $h_{h}$ and $h_{l}$ are the maximum and minimum thicknesses of the Gaussian-shaped foil, respectively.

\begin{tabular}{|c|c|c|c|c|c|c|c|c|}
\hline & $\begin{array}{c}\text { Intensity of } \\
\text { the laser }\end{array}$ & $\begin{array}{l}\text { Spot radius } \\
\text { of the laser }\end{array}$ & Half-aperture & $\begin{array}{l}\text { Thickness of } \\
\text { the cone wall }\end{array}$ & $\begin{array}{l}\text { Composition of } \\
\text { the cone wall }\end{array}$ & $\begin{array}{l}\text { Profile of the } \\
\text { cone tip }\end{array}$ & $\begin{array}{l}\text { Thickness of } \\
\text { the cone tip }\end{array}$ & $\begin{array}{l}\text { Composition of } \\
\text { the cone tip }\end{array}$ \\
\hline Case A & & & & & $\mathrm{Au}^{40+}+\mathrm{e}^{-}$ & Slab & $0.14 \lambda_{0}$ & \\
\hline Case B & $1.7 \times 10^{21} \mathrm{~W} / \mathrm{cm}^{2}$ & $13.5 \lambda_{0}$ & $20^{\circ}$ & $1.5 \lambda_{0}$ & $\mathrm{H}^{+}+\mathrm{e}^{-}$ & Slab & $0.14 \lambda_{0}$ & $\mathrm{C}^{6+}+\mathrm{Cu}^{24+}+\mathrm{e}^{-}$ \\
\hline Case C & & & & & $\mathrm{H}^{+}+\mathrm{e}^{-}$ & Gaussian-shaped & $h_{h}=0.2 \lambda_{0} h_{l}=0.1 \lambda_{0}$ & \\
\hline
\end{tabular}



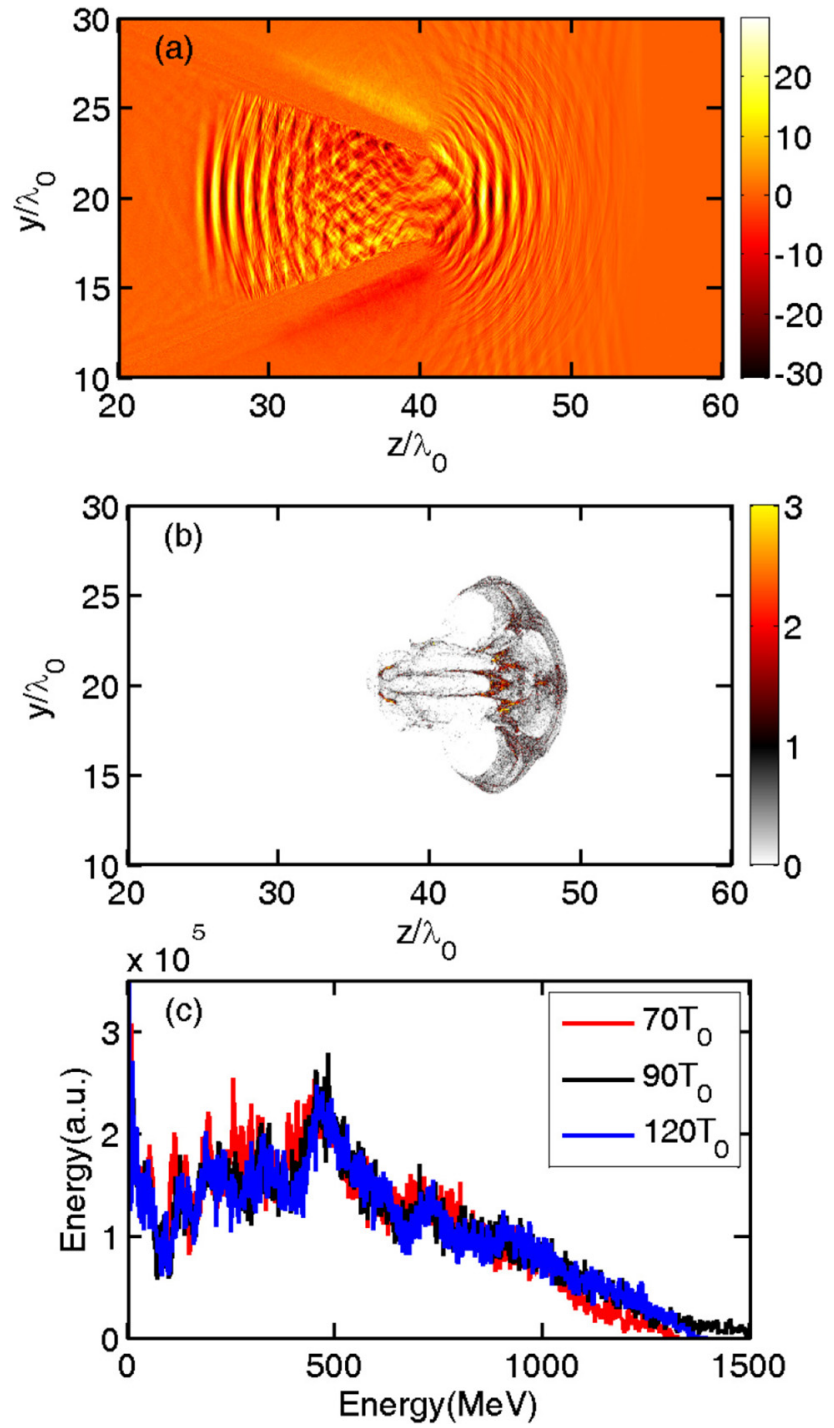

FIG. 2. Distribution of the transverse electric field $\left(E_{y}\right)$ at $\mathrm{t}=55 T_{0}$ (a) and of the charge density of $\mathrm{C}^{6+}$ ions at $\mathrm{t}=70 T_{0}$ (b) for case A. Energy spectra of the $\mathrm{C}^{6+}$ ions at $\mathrm{t}=70 T_{0}, \mathrm{t}=90 T_{0}$, and $120 T_{0}$ for case $\mathrm{A}(\mathrm{c})$. The electric field and density are expressed in units of $m_{e} c \omega_{0} / e$ and $n_{c}$, respectively (same as in the other figures).

dimensionless amplitude near the laser axis increases from $a_{0}=25$ (intensity at the entrance of the cone) to $a_{0}=50$ (intensity at the cone tip) at $t=50 T_{0}$, corresponding to a laser intensity of $6.85 \times 10^{21} \mathrm{~W} / \mathrm{cm}^{2}$, i.e., a 4-fold intensity enhancement. The enhanced laser intensity is now concentrated on a slab foil of $2 \lambda_{0}$ radius as compared with the original laser spot radius of $13.5 \lambda_{0}$.

When the laser impacts the slab foil, the radiation pressure at the foil center is much stronger than at the periphery. The slab foil is strongly deformed along the laser axis, until it disassembles. The corresponding $\mathrm{C}^{6+}$ ion distribution is shown in Fig. 2(b). As the laser pulse overtakes the fragmented foil along the laser axis, the longitudinal component acts on the fragments, while the radial component tends to impart transverse momentum to them. The laser pulse is modulated by the cone structure (see Fig. 2(a)). Figure 2(c) shows the spectra of $\mathrm{C}^{6+}$ ions at $t=70 T_{0}$ (red), 90T (black), and $120 T_{0}$ (blue). Note that all $\mathrm{C}^{6+}$ ion spectra in this paper are integrated over the whole simulation box. The other parameters are the same as in Figs. 2(a) and 2(b). It can be seen that the spectra evolve slowly after $t=90 T_{0}$. In addition to some peaks at lower energy, we observed energetic $\mathrm{C}^{6+}$ ions of energy $>500 \mathrm{MeV}$, which, as shown in Figure 2(c), have a monotonously decreasing distribution. Although the maximum energy of $\mathrm{C}^{6+}$ ions is $1.5 \mathrm{GeV}$ as seen in Fig. 2(c), the number of ions at this energy is extremely small (only $\sim 0.4 \%$ of the total $\mathrm{C}^{6+}$ ions). We note that in case $\mathrm{A}$, the gold plasma cone wall stays intact during the laser interaction with the cone, as shown by Fig. 3(a), at $t=65 T_{0}$. It is clear that the approach of case A is not a promising one, if the objective is to obtain an ion beam with high energy density and a narrow-band spectral distribution for $\mathrm{C}^{6+}$ ions.

Prior publications have shown that it is possible to stabilize the radiation pressure drive of a compressed foil if the pulse can wrap around the plasma. ${ }^{31,39}$ For this purpose, a hydrogen cone is used in case B instead of the gold cone. The cone has a wall density of $50 n_{c}$, while the other parameters for the laser and the foil are the same as that in case A. Figure 3(b) shows the cone charge density at $t=65 T_{0}$ after laser irradiation. The H-plasma wall is broken near the tip, with small radiation leaks in these regions. Figure 4 shows the distribution of the transverse electric field $\left(E_{y}\right)(\mathrm{a})$, the charge density (b), and the energy spectrum (c) of $\mathrm{C}^{6+}$ ions for case B. The pulse here can be transmitted not only as a result of foil fragmentation but also through the broken cone wall. As a result, the laser electric field distribution around the cone tip becomes more uniform compared to that of case A. We note that, as the laser penetrates through the broken cone wall and propagates outward, it wraps around the foil clumps near the tip, and the radial radiation pressure tends to

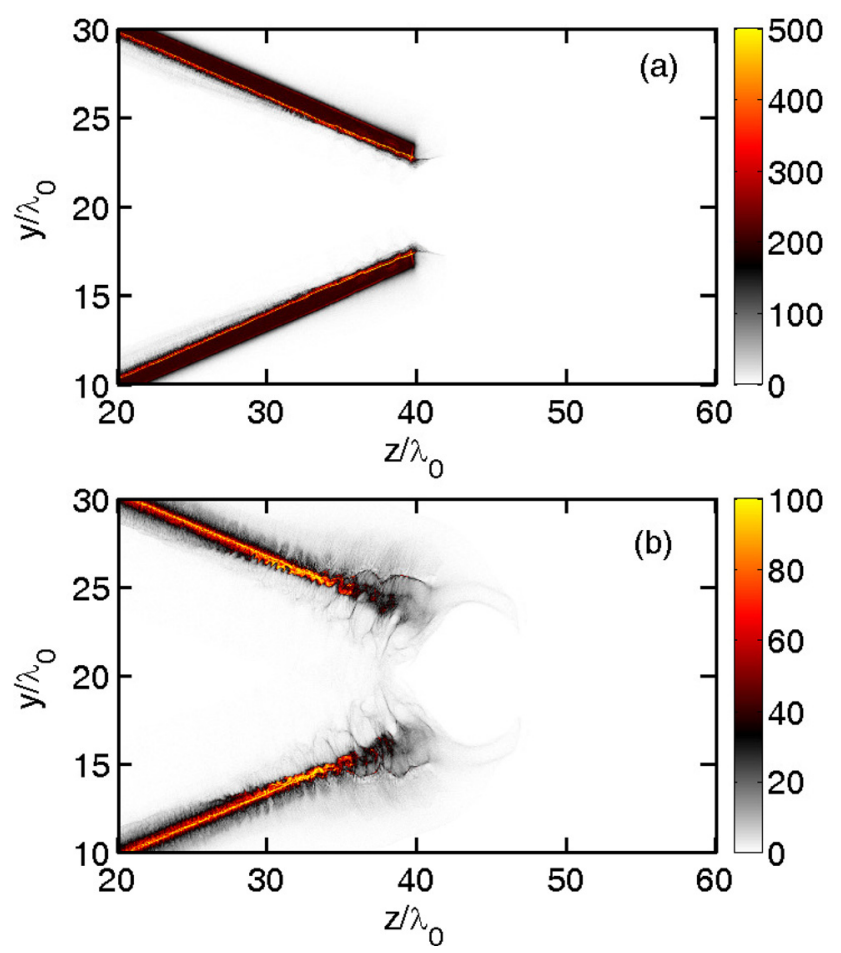

FIG. 3. Charge density distributions of the $\mathrm{Au}^{40+}$ ions at $\mathrm{t}=65 T_{0}$ for case $\mathrm{A}$ (a) and protons for case B (b), respectively. 

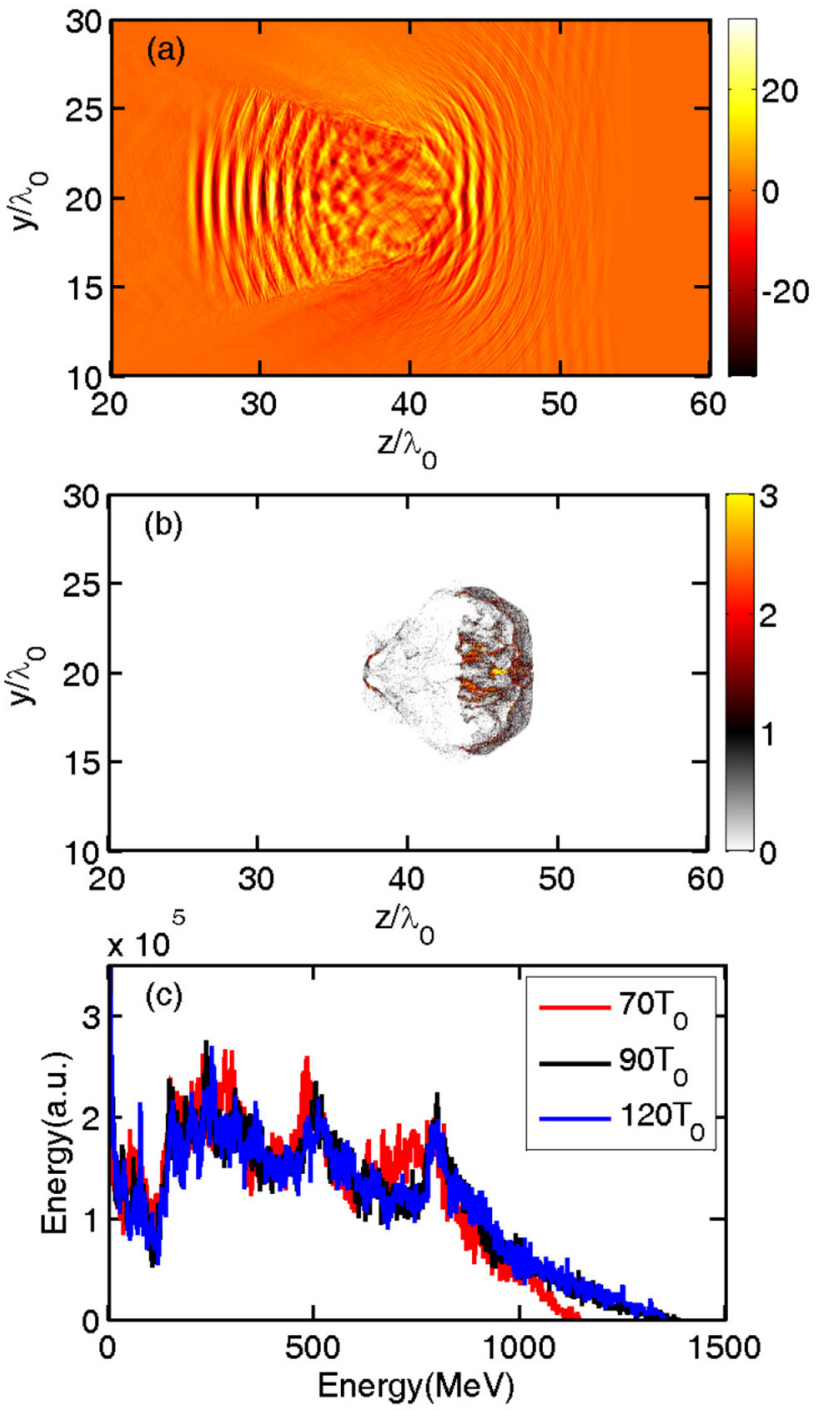

FIG. 4. Distribution of the transverse electric field at $\mathrm{t}=55 T_{0}$ (a) and of the charge density of $\mathrm{C}^{6+}$ ions at $\mathrm{t}=70 T_{0}$ (b) for case B. Energy spectra of the $\mathrm{C}^{6+}$ ions at $\mathrm{t}=70 T_{0}, \mathrm{t}=90 T_{0}$, and $120 T_{0}$ for case $\mathrm{B}(\mathrm{c})$.

stabilize, to some extent, their acceleration. One can see that, in Fig. 4(b), the density distribution of the $\mathrm{C}^{6+}$ ions is more localized than in Fig. 2(b). Fig. 4(c) shows that the distribution of $\mathrm{C}^{6+}$ ions with energies $>500 \mathrm{MeV}$ is no longer monotonously decreasing and that a peak emerges in the spectrum near $800 \mathrm{MeV}$. However, this peak is not very pronounced and only contains a small number of $\mathrm{C}^{6+}(\sim 10 \%$ of the total $\mathrm{C}^{6+}$ ions).

To further improve the quality of the laser-accelerated $\mathrm{C}^{6+}$ ion bunches, we consider a different target design in case $\mathrm{C}$, which is composed of a hydrogen plasma cone and a Gaussian-shaped foil at the cone tip, as suggested in Ref. 16. The composition of the Gaussian-shaped foil is identical to that of cases $\mathrm{A}$ and $\mathrm{B}$. The thickness of the foil is given by $h=\max \left\{h_{h} \times \exp \left(-\left(y-20 \lambda_{0}\right) / y_{0}^{2}\right), h_{l}\right\}$, where $y_{0}=2 \lambda_{0}$ is the spot radius and $h_{h}=0.2 \lambda_{0}$ and $h_{l}=0.1 \lambda_{0}$ are the maximum and minimum thicknesses of the Gaussian-shaped foil, as shown in Fig. 1(b).

At the center of the Gaussian-shaped foil, where both the foil thickness and the laser intensity are at their maximum, the multi-species foil is effectively accelerated as a plasma bunch along the laser axis. The laser can escape the cone from breaks in the H-plasma cone wall, as well as from the thinner periphery of the Gaussian-shaped foil. The transmitted radiation then enfolds the plasma layer accelerated from the cone tip with beneficial effects toward its stabilization, as discussed in Ref. 30. We note that the electric field distribution in case $\mathrm{C}$ is obviously different from that of case A, i.e., the electric field at the center of the foil is weaker than that at the periphery. Moreover, in case $\mathrm{C}$, the accelerated and compressed $\mathrm{C}^{6+}$ ions concentrate along the laser axis, with densities up to $3 n_{c}$ at $t=70 T_{0}$, which is much higher than that of cases A and B. Since our current PIC code does not have the capability of tracking the particles, here we do not track the particles of the bunches. The $\mathrm{Cu}^{24+}$ ions spread widely in space and do not form a clear bunch, whose energy spectra are monotonously decreasing. Figure 5(c) shows the spectrum of the $\mathrm{C}^{6+}$ ions. The most prominent feature here is the quasi-mono-energetic peak at
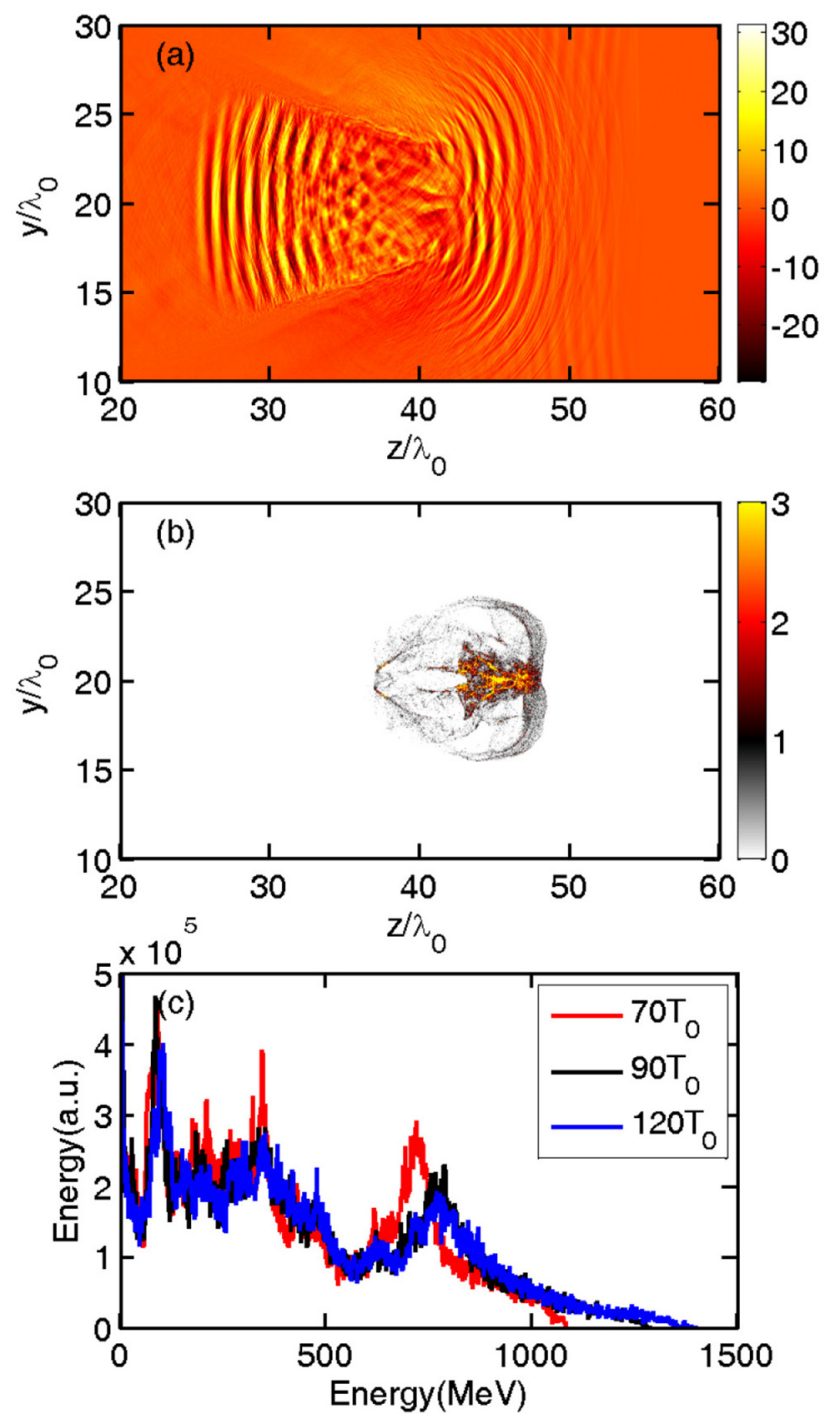

FIG. 5. Distribution of the transverse electric field at $\mathrm{t}=55 T_{0}$ (a) and of the charge density of $\mathrm{C}^{6+}$ ions at $\mathrm{t}=70 T_{0}$ (b) for case $\mathrm{C}$. Energy spectra of the $\mathrm{C}^{6+}$ ions at $\mathrm{t}=70 T_{0}, \mathrm{t}=90 T_{0}$, and $120 T_{0}$ for case $\mathrm{C}(\mathrm{c})$. 
$\sim 720 \mathrm{MeV}$, which contains about $37 \%$ of the total $\mathrm{C}^{6+}$ ion energy at $t=70 T_{0}$. The energy spread of the peak is $\Delta E / E \sim 14 \%$. The energy of this peak increases to $790 \mathrm{MeV}$ at $t=90 T_{0}$, when $31 \%$ of the total $\mathrm{C}^{6+}$ ion energy is contained in the peak. It is noted that the ion bunch can maintain the quasi-mono-energetic peak for a long period $\left(\Delta t>80 T_{0}\right)$ after the laser is turned off. The ions contained in the peak leave the simulation box at $\mathrm{t}=140 \mathrm{~T}_{0}$.

Figure 6(a) shows the distribution of the kinetic energy density of the $\mathrm{C}^{6+}$ ions at $t=70 T_{0}$. It is shown that the accelerated ion bunch has a very high energy density $\left(\sim 7 \times 10^{10} \mathrm{~J} / \mathrm{cm}^{3}\right)$, which is comparable to the energy density of the incident laser pulse $\left(\sim 10^{11} \mathrm{~J} / \mathrm{cm}^{3}\right)$. For comparison, in Fig. 6(b), we also show the kinetic energy density distribution of the $\mathrm{C}^{6+}$ for case $\mathrm{A}$. In this case, the ions become very divergent, leading to the energy density around the laser propagation axis being significantly lower than that in Fig. 6(a). Note that LAPINE is a very efficient PIC code, and both the initial amount of energy and the effect of numerical heating are negligible compared to the energy from the laser (i.e., only $0.05 \%$ of the energy from the laser).

Figure 7 shows the time evolution of the total energy of the $\mathrm{C}^{6+}$ ions in case $\mathrm{C}$. One can see that the total energy grows rapidly as soon as the laser pulse impacts the Gaussian-shaped foil. The energy keeps growing at later times, as the laser penetrates the cone from the foil periphery and wraps around the accelerated ion bunch. The energy conversion efficiency from laser to $\mathrm{C}^{6+}$ ions is $\sim 4 \%$, and about $1.5 \%$ laser energy is contained in the $720 \mathrm{MeV}$ quasimono-energetic peak at $t=70 T_{0}$.
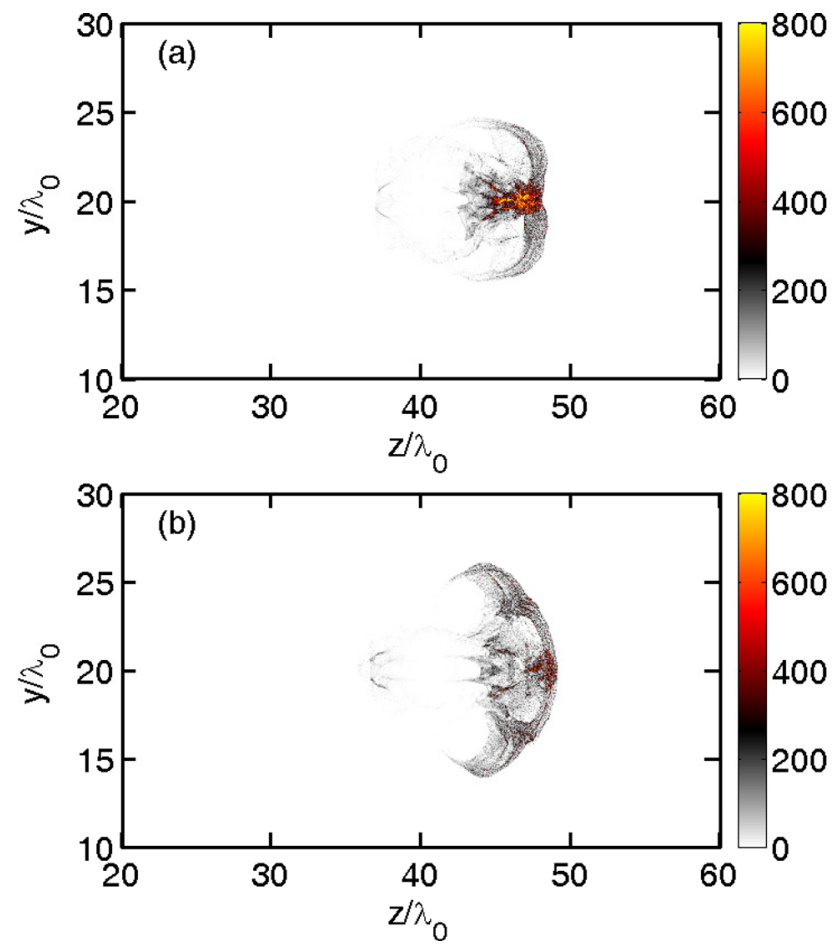

FIG. 6. Kinetic energy density distribution of the $\mathrm{C}^{6+}$ ions at $\mathrm{t}=70 T_{0}$ for cases C (a) and A (b), respectively. The kinetic energy density is expressed in units of $m_{e} c^{2} n_{c}$.

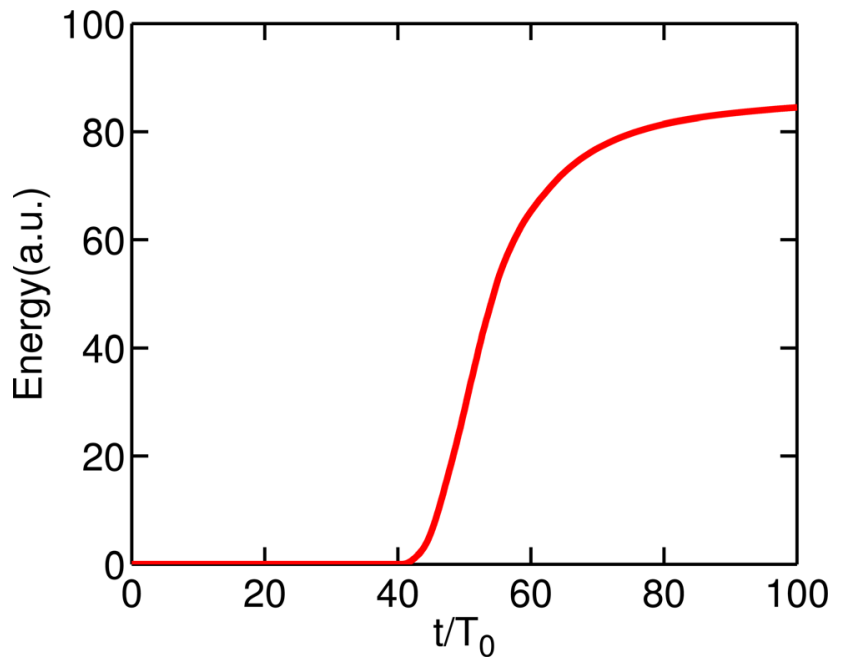

FIG. 7. Total energy of the $\mathrm{C}^{6+}$ ions as a function of time for case C.

For completeness, we also study the cases of a slab foil only and with dimensionless amplitudes $a_{0}=25$ and 50, respectively. The foil here has the same composition and thickness (i.e., $0.14 \lambda_{0}$ ) as that of the tip of case A. The charge density distributions and energy spectra of the $\mathrm{C}^{6+}$ ions for the two cases are presented in Fig. 8. It can be seen that, in Fig. 8(a), the foil is not broken up by the laser pulse due to the relatively lower laser intensity. The corresponding energy spectra are monotonously decreasing (see Fig. 8(c)), whose cutoff energies are only $\sim 170 \mathrm{MeV}$, significantly lower than that of case $\mathrm{C}$. As the laser intensity increases to the focused intensity $\left(a_{0}=50\right)$, one can see that the laser pulse can greatly push forward the foil target and drive the $\mathrm{C}^{6+}$ ions to higher energies, as shown in Figs. 8(b) and 8(d). However, there is also no obvious energy peak appearing in the spectra.

Due to the limited computing resources, it is impossible to simulate the whole distance of laser ion acceleration and following propagation. Despite the fact that only a small region including the cone and tip was simulated, the enhanced effect of the cone on the laser intensity and the optimized effect on the following ion acceleration can be observed well. From the simulations, we note that the spectrum of ions becomes quasi-mono-energetic rapidly during the laser interaction and evolves slowly after the laser is turned off. Such ion bunches might spread widely at a much later time due to the loss of the electrons. However, several schemes have been proposed to further enhance the stability and reduce the divergence of high-energy-density ion bunches during the long distance propagation, such as using a background plasma to partially neutralize the spacecharge $^{40}$ or using an extra laser-driven micro-lens to control the emission of protons. ${ }^{41}$

Considering a realistic 3D cone target, the laser pulse would be focused to a much tighter spot due to the fact that the laser intensity at the cone tip is $\propto I\left(r_{0} / r_{t}\right)^{2}$, where $r_{0}$ and $r_{t}$ are the inner radius of the cone hatch and tip, respectively. Therefore, ion bunches with a much higher energy and density might be obtained employing our scheme with a less intense laser pulse, which would have to be examined in future studies. 

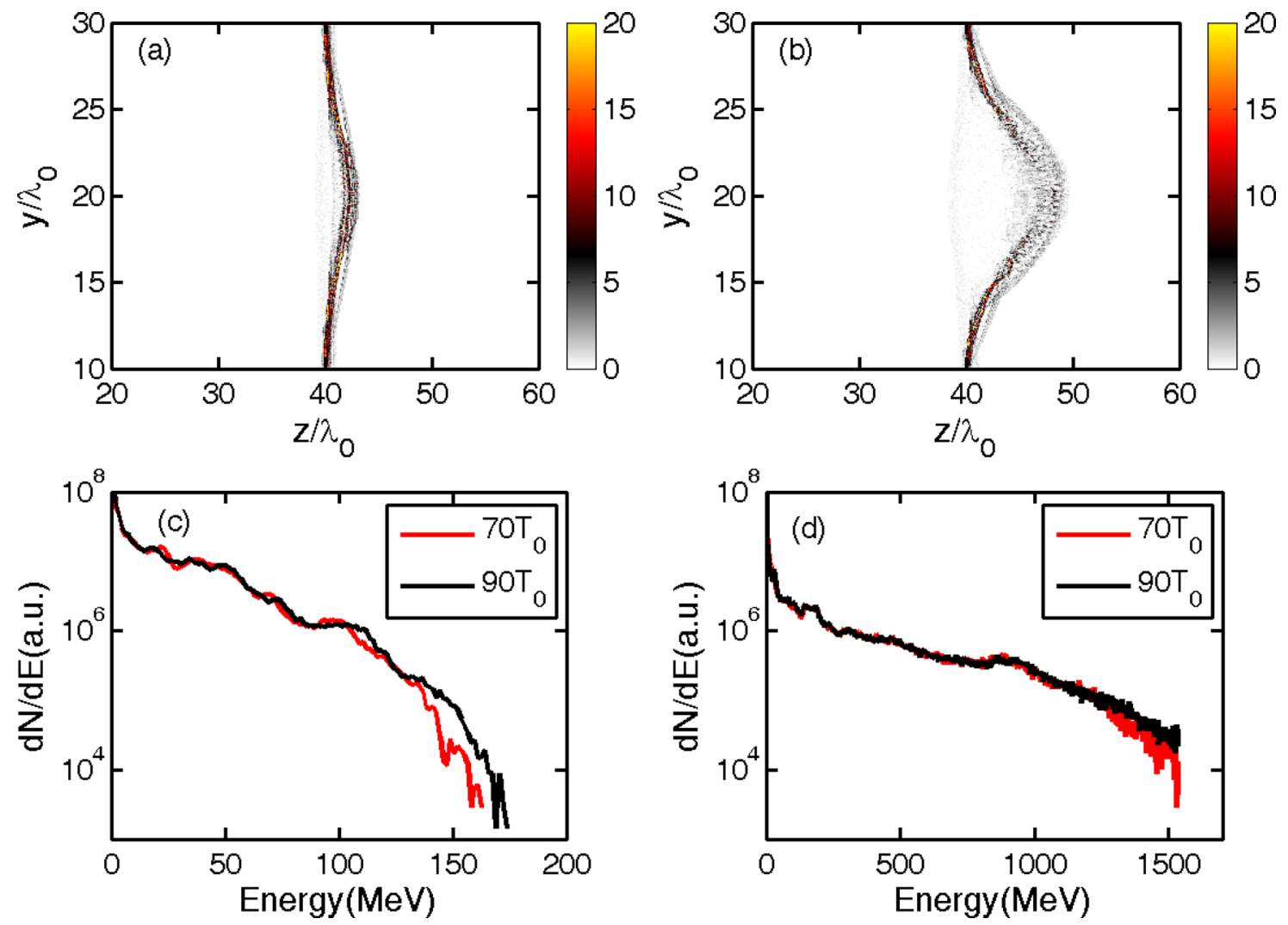

FIG. 8. Distribution of the charge density of $\mathrm{C}^{6+}$ ions at $\mathrm{t}=70 T_{0}$ for the cases of a slab foil only and with $a_{0}=25$ (a) and 50 (b), respectively. Energy spectra of the $\mathrm{C}^{6+}$ ions at $\mathrm{t}=70 T_{0}$ and $90 T_{0}$ for the cases of a slab foil only and with $a_{0}=25$ (c) and 50 (d), respectively. The other parameters are the same as that in case $\mathrm{C}$.

\section{CONCLUSION}

In summary, we have examined intense laserirradiation, with circularly polarized pulses, of a number of cone-foil target configurations, with the aim of identifying possible combinations suitable for producing high-energy, narrow-band carbon ions. In particular, we have searched for configurations in which the intensity enhancement determined by pulse constriction within the cone can be used to efficiently drive the RPA of an ultrathin foil. We found an interesting configuration by combining a hydrogen cone with a multispecies foil with thickness varying radially according to a Gaussian function (as suggested in prior publication ${ }^{16}$ ). A narrow-band, high energy density $\mathrm{C}^{6+}$ ion bunch, with a peak energy of $790 \mathrm{MeV}$, results from the combination of a number of factors which provide a stable and efficient acceleration of the carbon ion layer: (1) intensity (and radiation pressure) enhancement due to cone guiding; (2) matching of the foil thickness to the intensity profile ${ }^{16}$ (3) multispecies effects; ${ }^{21,22}$ and (4) enfolding of the accelerated clump by transmitted radiation. ${ }^{30}$

\section{ACKNOWLEDGMENTS}

This work was supported by the NSFC (Grant Nos. 11305264, 11275269, 11175253, 11375265, and 91230205), the RFDP (Grant No. 20114307110020), the Open Fund of the State Key Laboratory of High Field Laser Physics at SIOM, and the Research Program of NUDT. M.B. acknowledges funding from projects ELI (Grant No. CZ.1.05/1.1.00/483/02.0061) and OPVK 3 (Grant No. CZ.1.07/2.3.00/20.0279).

${ }^{1}$ M. Roth, T. E. Cowan, M. H. Key, S. P. Hatchett, C. Brown, W. Fountain, J. Johnson, D. M. Pennington, R. A. Snavely, S. C. Wilks, K. Yasuike, H. Ruhl, F. Pegoraro, S. V. Bulanov, E. M. Campbell, M. D. Perry, and H. Powell, Phys. Rev. Lett. 86, 436 (2001).

${ }^{2}$ E. Fourkal, V. Velchev, J. Fan, W. Luo, and C. M. Ma, Med. Phys. 34, 577 (2007).

${ }^{3}$ M. Borghesi, S. V. Bulanov, T. Zh. Esirkepov, S. Fritzler, S. Kar, T. V. Liseikina, V. Malka, F. Pegoraro, L. Romagnani, J. P. Rousseau, A. Schiavi, O. Willi, and A. V. Zayats, Phys. Rev. Lett. 94, 195003 (2005).

${ }^{4}$ G. Sarri, A. Macchi, C. A. Cecchetti, S. Kar, T. V. Liseykina, X. H. Yang, M. E. Dieckmann, J. Fuchs, M. Galimberti, L. A. Gizzi, R. Jung, I. Kourakis, J. Osterholz, F. Pegoraro, A. P. L. Robinson, L. Romagnani, O. Willi, and M. Borghesi, Phys. Rev. Lett. 109, 205002 (2012).

${ }^{5}$ S. C. Wilks, A. B. Langdon, T. E. Cowan, M. Roth, M. Singh, S. Hatchett, M. H. Key, D. Pennington, A. MacKinnon, and R. A. Snavely, Phys. Plasmas 8, 542 (2001)

${ }^{6}$ L. Robson, P. T. Simpson, R. J. Clarke, K. W. D. Ledingham, F. Lindau, O. Lundh, T. McCanny, P. Mora, D. Neely, C.-G. Wahlström, M. Zepf, and P. McKenna, Nature Phys. 3, 58 (2007).

${ }^{7}$ L. Yin, B. J. Albright, B. M. Hegelich, and J. C. Fernández, Laser Part. Beams 24, 291 (2006).

${ }^{8}$ A. Macchi, F. Cattani, T. V. Liseykina, and F. Cornolti, Phys. Rev. Lett. 94, 165003 (2005).

${ }^{9}$ H. B. Zhuo, Z. L. Chen, W. Yu, Z. M. Sheng, M. Y. Yu, Z. Jin, and R. Kodama, Phys. Rev. Lett. 105, 065003 (2010).

${ }^{10}$ T. Ceccotti, A. Lévy, H. Popescu, F. Réau, P. D’Oliveira, P. Monot, J. P. Geindre, E. Lefebvre, and Ph. Martin, Phys. Rev. Lett. 99, 185002 (2007).

${ }^{11}$ Y. Y. Ma, Z. M. Sheng, Y. Q. Gu, M. Y. Yu, Y. Yin, F. Q. Shao, T. P. Yu, and W. W. Chang, Phys. Plasmas 16, 034502 (2009).

${ }^{12}$ P. Mora, Phys. Rev. E 72, 056401 (2005). 
${ }^{13}$ H. Daido, M. Nishiuchi, and A. S. Pirozhkov, Rep. Prog. Phys. 75, 056401 (2012).

${ }^{14}$ X. Zhang, B. F. Shen, X. M. Li, Z. Y. Jin, F. C. Wang, and M. Wen, Phys. Plasmas 14, 123108 (2007).

${ }^{15}$ X. Q. Yan, C. Lin, Z. M. Sheng, Z. Y. Guo, B. C. Liu, Y. R. Lu, J. X. Fang, and J. E. Chen, Phys. Rev. Lett. 100, 135003 (2008).

${ }^{16}$ M. Chen, A. Pukhov, T. P. Yu, and Z. M. Sheng, Phys. Rev. Lett. 103, 024801 (2009).

${ }^{17}$ Z. M. Zhang, X. T. He, Z. M. Sheng, and M. Y. Yu, Appl. Phys. Lett. 100, 134103 (2012).

${ }^{18}$ A. Macchi, S. Veghini, and F. Pegoraro, Phys. Rev. Lett. 103, 085003 (2009).

${ }^{19}$ B. Qiao, M. Zepf, M. Borghesi, and M. Geissler, Phys. Rev. Lett. 102, 145002 (2009).

${ }^{20}$ See http://www.extreme-light-infrastructure.eu for the Extreme Light Infrastructure European project (ELI).

${ }^{21}$ T. P. Yu, A. Pukhov, G. Shvets, and M. Chen, Phys. Rev. Lett. 105, 065002 (2010).

${ }^{22}$ B. Qiao, M. Zepf, P. Gibbon, M. Borghesi, B. Dromey, S. Kar, J. Schreiber, and M. Geissler, Phys. Plasmas 18, 043102 (2011).

${ }^{23}$ W. Zhou, Y. Gu, W. Hong, L. Cao, Z. Zhao, Y. Ding, B. Zhang, H. Cai, and K. Mima, Laser Part. Beams 28, 585 (2010).

${ }^{24}$ X. H. Yang, H. Xu, Y. Y. Ma, F. Q. Shao, Y. Yin, H. B. Zhuo, M. Y. Yu, and C. L. Tian, Phys. Plasmas 18, 023109 (2011).

${ }^{25}$ L. H. Cao, W. Yu, M. Y. Yu, H. Xu, X. T. He, Y. Q. Gu, Z. J. Liu, J. H. Li, and C. Y. Zheng, Phys. Rev. E 78, 036405 (2008).

${ }^{26}$ K. A. Flippo, E. d'Humières, S. A. Gaillard, J. Rassuchine, D. C. Gautier, M. Schollmeier, F. Nürnberg, J. L. Kline, J. Adams, B. Albright, M. Bakeman, K. Harres, R. P. Johnson, G. Korgan, S. Letzring, S. Malekos, N. Renard-LeGalloudec, Y. Sentoku, T. Shimada, M. Roth, T. E. Cowan, J. C. Fernández, and B. M. Hegelich, Phys. Plasmas 15, 056709 (2008).

${ }^{27}$ S. A. Gaillard, T. Kluge, K. A. Flippo, M. Bussmann, B. Gall, T. Lockard, M. Geissel, D. T. Offermann, M. Schollmeier, Y. Sentoku, and T. E. Cowan, Phys. Plasmas 18, 056710 (2011).

${ }^{28}$ T. Kluge, S. A. Gaillard, K. A. Flippo, T. Burris-Mog, W. Enghardt, B. Gall, M. Geissel, A. Helm, S. D. Kraft, T. Lockard, J. Metzkes, D. T.
Offermann, M. Schollmeier, U. Schramm, K. Zeil, M. Bussmann, and T. E. Cowan, New J. Phys. 14, 023038 (2012).

${ }^{29}$ B. Qiao, S. Kar, M. Geissler, P. Gibbon, M. Zepf, and M. Borghesi, Phys. Rev. Lett. 108, 115002 (2012).

${ }^{30}$ X. Q. Yan, H. C. Wu, Z. M. Sheng, J. E. Chen, and J. Meyer-ter-Vehn, Phys. Rev. Lett. 103, 135001 (2009).

${ }^{31}$ H. Xu, W. Yu, M. Y. Yu, A. Y. Wong, Z. M. Sheng, M. Murakami, and J. Zhang, Appl. Phys. Lett. 100, 144101 (2012).

${ }^{32}$ X. H. Yang, M. E. Dieckmann, G. Sarri, and M. Borghesi, Phys. Plasmas 19, 113110 (2012).

${ }^{33}$ J. Fuchs, P. Antici, E. D’Humiéres, E. Lefebvre, M. Borghesi, E. Brambrink, C. A. Cecchetti, M. Kaluza, V. Malka, M. Manclossi, S. Meyroneinc, P. Mora, J. Schreiber, T. Toncian, H. Pépin, and P. Audebert, Nature Phys. 2, 48 (2006).

${ }^{34}$ R. C. Shah, R. P. Johnson, T. Shimada, K. A. Flippo, J. C. Fernandez, and B. M. Hegelich, Opt. Lett. 34, 2273 (2009).

${ }^{35}$ A. Jullien, O. Albert, F. Burgy, G. Hamoniaux, J. Rousseau, J. Chambaret, F. Augé-Rochereau, G. Chériaux, J. Etchepare, N. Minkovski, and S. M. Saltiel, Opt. Lett. 30, 920 (2005).

${ }^{36}$ A. Jullien, J. Rousseau, B. Mercier, L. Antonucci, O. Albert, G. Chériaux, S. Kourtev, N. Minkovski, and S. M. Saltiel, Opt. Lett. 33, 2353 (2008).

${ }^{37}$ A. Yogo, K. Kondo, M. Mori, H. Kiriyama, K. Ogura, T. Shimomura, N. Inoue, Y. Fukuda, H. Sakaki, S. Jinno, M. Kanasaki, and P. R. Bolton, Opt. Express 22, 2060 (2014).

${ }^{38}$ G. Doumy, F. Quéré, O. Gobert, M. Perdrix, Ph. Martin, P. Audebert, J. C. Gauthier, J. P. Geindre, and T. Wittmann, Phys. Rev. E 69, 026402 (2004).

${ }^{39}$ W. Yu, H. Xu, F. He, M. Y. Yu, S. Ishiguro, J. Zhang, and A. Y. Wong, Phys. Rev. E 72, 046401 (2005).

${ }^{40}$ I. D. Kaganovich, E. A. Startsev, A. B. Sefkow, and R. C. Davidson, Phys. Plasmas 15, 103108 (2008).

${ }^{41}$ T. Toncian, M. Borghesi, J. Fuchs, E. d'Humières, P. Antici, P. Audebert, E. Brambrink, C. A. Cecchetti, A. Pipahl, L. Romagnani, and O. Willi, Science 312, 410 (2006). 